\title{
Immunohistochemical analysis of matrix metalloproteinase-9 predicts papillary thyroid carcinoma prognosis
}

\author{
XINGKAI LIU ${ }^{1 *}$, CHANG SU$^{2 *}$, JING XU $^{3 *}$, DAN ZHOU $^{4}$, HE YAN $^{5}$, WEI LI $^{5}$, \\ GUIHUI CHEN $^{6}$, NAN ZHANG ${ }^{5}$, DAHAI XU ${ }^{5}$ and HAIXIA HU ${ }^{5}$ \\ Departments of ${ }^{1}$ Hepatobiliary and Pancreatic Surgery, and ${ }^{2}$ Thyroid Surgery; ${ }^{3}$ Cardiovascular Disease Center, \\ The First Hospital of Jilin University, Changchun, Jilin 130021; ${ }^{4}$ Department of Pediatrics, The Second Hospital of \\ Jilin University, Changchun, Jilin 130041; ${ }^{5}$ Department of Emergency, The First Hospital of Jilin University, Changchun, \\ Jilin 130021; ${ }^{6}$ Department of Pathology, Jilin City People's Hospital, Jilin, Jilin 132000, P.R. China
}

Received March 24, 2018; Accepted November 14, 2018

DOI: $10.3892 / \mathrm{ol} .2018 .9850$

\begin{abstract}
The present study aimed to explore the association between immunohistochemical matrix metalloproteinase-9 (MMP-9) expression and the clinicopathological characteristics of patients with papillary thyroid carcinoma (PTC), and to determine whether it may be used as a diagnostic or prognostic tool for PTC. Immunohistochemical staining of MMP-9 was performed in thyroid tissues obtained from 112 patients with PTC and 42 subjects with benign thyroid nodules (BTNs). The receiver operating characteristic curve was used to evaluate the legitimacy of MMP-9 as a diagnostic tool for PTC, and a predictor for structurally persistent/recurrent disease (SPRD) and disease status. Cox regression was applied to identify the risk factors of disease status and SPRD. The present study revealed that MMP-9 was overexpressed in PTC tissues, compared with in BTN tissues. Furthermore, MMP-9 scores yielded an area under the curve (AUC) of 0.842 (95\% CI, 0.776-0.908) for differentially diagnosing PTC from BTN. In addition, the MMP-9 score was greater if patients previously had central lymph node metastasis, lateral lymph node metastasis or an advanced tumor-node-metastasis stage (III+IV). When MMP-9 was employed to predict disease status and SPRD, an AUC of 0.811 (95\% CI, 0.706-0.917) and 0.806 (95\% CI, 0.620-0.992) was obtained, respectively. A tumor size of $>2 \mathrm{~cm}$ and an MMP-9 staining score of $\geq 6$ were independent risk factors for predicting disease status, whereas
\end{abstract}

Correspondence to: Dr Dahai Xu or Dr Haixia Hu, Department of Emergency, The First Hospital of Jilin University, 71 Xinmin Street, Changchun, Jilin 130021, P.R. China

E-mail: yangzhouxux@sina.com

E-mail: 504873697@qq.com

*Contributed equally

Key words: matrix metalloproteinase-9, papillary thyroid carcinoma, disease-free survival, structurally persistent/recurrent disease, prognosis vascular invasion and an MMP-9 staining score of $\geq 8$ were risk factors for predicting SPRD. Furthermore, an MMP-9 staining score of $\geq 6$ and $\geq 8$ indicated shortened disease-free survival and survival without SPRD, respectively. In conclusion, the assessment of MMP-9 expression in thyroid carcinoma samples may represent a potential and supplementary tool for the diagnosis and prognostic prediction of PTC.

\section{Introduction}

The incidence of thyroid carcinoma has markedly increased worldwide in the past 4 decades $(1,2)$. Papillary thyroid carcinoma (PTC) represents the most common type of well-differentiated thyroid cancer, accounting for $80-85 \%$ of thyroid malignancies (3). Patients with PTC have a generally favorable prognosis (2); however, a 30-year follow-up study demonstrated that recurrence rates and cancer mortality are high in PTC patients with local tumor invasion and lymph node metastasis (LNM) (4). Although the predictive value of several biomarkers for PTC prognosis, including microRNA-451 (5), B-Raf proto-oncogene, serine/threonine kinase mutation (6) and EH domain-containing 2 (7) have been investigated, their efficacy has yet to be validated. To the best of our knowledge, reliable biomarkers able to predict the invasiveness of PTC and prognosis of patients are still lacking.

Matrix metalloproteinases (MMPs) are a family of zinc-dependent extracellular proteases that maintain and remodel tissue architecture. The degradation of basement membranes and of extracellular matrix (ECM) is an essential step in tumor invasion and migration; therefore, MMPs serve an essential role in cancer metastasis $(8,9)$. MMP-9, also known as $92 \mathrm{kDa}$ gelatinase/type IV collagenase, is a prominent MMP that is responsible for the migratory and invasive abilities of diverse types of cancer (10-16). MMPs are closely involved in the stimulation of angiogenesis, which is essential for tumor growth and progression (17). In addition, MMP-9 facilitates the release of tissue-bound fibroblast growth factor (FGF) $(18,19)$ and vascular endothelial growth factor (VEGF), which contribute to tumor growth. Consequently, MMP-9 may be considered a novel biomarker and a potential therapeutic target in human cancer. 
Considering its contribution to cancer initiation, tumor growth, angiogenesis and migration, the diagnostic and prognosis capacities of MMP-9 have been assessed in various types of cancer $(16,20,21)$. Furthermore, the overexpression of MMP-9 in colorectal cancer tissue is associated with tumor invasion, LNM and advanced tumor-node-metastasis (TNM) stage (20). In addition, increased expression of MMP-9 is observed in patients with glioma, which is associated with advanced glioma grades and negative survival rates (21). Researchers have speculated that MMP-9 overexpression promotes circulating tumor cells to shed into the bloodstream by breaking the basement membrane or digesting ECM (16).

As determined by immunohistochemistry (IHC), MMP-9 is involved in the progression and aggressiveness of PTC, although some inconsistencies regarding its predictive capacity have been reported (22-24). The present study aimed to investigate the expression of MMP-9 in tumors from patients with PTC in comparison with samples obtained from patients with benign thyroid nodules (BTNs). The association between MMP expression and the clinicopathological characteristics of patients with PTC was also analyzed. Eventually, the diagnostic and prognostic value of immunohistochemical MMP-9 expression for PTC was investigated.

\section{Patients and methods}

Study population. A group of patients with PTC was retrospectively reviewed at the First Hospital of Jilin University between January 1, 2012 and June 31, 2014. Furthermore, age- and sex-matched subjects with BTN were recruited as controls. The diagnosis of PTC and BTN was made on a pathological basis. BTN was defined as follows: A lump inside the thyroid, an up and down movement when swallowing, and a biopsy confirming the benign nature of the lump. BTNs included nodular goiter and thyroid adenoma. The inclusion criteria for PTC or BTNs were as follows: i) biopsy-confirmed PTC or BTN; ii) age ranged from 18-70 years old. The exclusion criteria for PTC and BTNs were as follows: i) Anticancer therapy prior to admission, including radiotherapy, chemotherapy and thyroidectomy; ii) severe systemic disorders, including heart failure and malignancies; iii) incomplete or missing test results. The present study was approved by the Ethics Committee of the First Hospital of Jilin University, and all participants provided informed consent prior to participation.

The body mass index (BMI) of each patient was calculated as the weight in kilograms divided by height in meters squared $\left(\mathrm{kg} / \mathrm{m}^{2}\right)$. All patients with PCT were graded at the time of diagnosis, according to the 7 th edition of the American Joint Committee on Cancer TNM staging system (25). Early-stage disease was named as TNM stage I or II, whereas advanced-stage disease was named as stage III or IV.

Thyroid surgery and follow-up. Bilateral or unilateral central-compartment neck dissection was routinely performed in patients with PTC, followed by total or partial thyroidectomy. In addition, patients underwent lateral neck lymph node dissection when the lateral cervical lymph nodes were suspected to be metastatic, based on explicit clinical and/or imaging findings. The same team of surgeons performed all surgical procedures.

Patients with PTC were followed up every 3 months until December 2017. All patients were monitored for postoperative PTC relapses by ultrasound, computed tomography or chest $\mathrm{X}$-ray, and in combination with biochemical measurements, including serum thyroglobulin ( $\mathrm{Tg}$ ) and $\mathrm{Tg}$ antibody ( $\mathrm{Tg} \mathrm{Ab}$ ). Disease status was defined as any evidence of disease, including persistently detectable $\mathrm{Tg} \mathrm{Ab}, \mathrm{Tg} \geq 1 \mathrm{ng} / \mathrm{ml}$, and structurally persistent disease or metastasis. Structurally persistent disease was defined as the occurrence of locoregional or distant metastasis, regardless of the Tg level. A recurrence event was defined as the structural evidence of a disease identified following a period of no evidence of disease. Disease-free status was classified as no evidence of disease, as aforementioned. Disease-free survival (DFS) time was defined as the period from initial therapy to any evidence of disease or last follow-up. Survival time without structurally persistent disease/recurrent disease (SPRD) was calculated as the interval from initial treatment to the occurrence of structurally persistent disease, recurrent disease or last follow-up.

Pathological subtypes by hematoxylin and eosin staining $(H \& E)$. Pathological subtypes of enrolled subjects with PTC were investigated by H\&E staining for $20 \mathrm{~min}$ at room temperature. The results were visualized by Olympus BX51 microscope (Olympus Corporation, Tokyo, Japan).

Determination of tissue MMP-9 expression by IHC. Thyroid neoplastic tissues were collected from patients with PTC or BTN during thyroidectomy. Tissue specimens were fixed in $4 \%$ paraformaldehyde in TBS at $4^{\circ} \mathrm{C}$ for $18 \mathrm{~h}$. Paraffin-embedded tissue specimens were sectioned at a thickness of $2.0 \mu \mathrm{m}$, followed by deparaffinization in xylene and dehydration in a graded series of ethanol solutions. Antigen retrieval was performed by heating the samples (in rice cooker for $20 \mathrm{~min}$ at $95^{\circ} \mathrm{C}$ ) in citrate buffer ( $\mathrm{pH}$ 6.0), and endogenous peroxidase was blocked with $3 \%$ hydrogen peroxide for $10 \mathrm{~min}$ at room temperature. Afterwards, the sections were incubated with rabbit polyclonal MMP-9 antibody (cat. no. ab38898; Abcam, Cambridge, MA, USA) at a dilution of $1: 250$ at $4^{\circ} \mathrm{C}$ overnight. After washing with PBS, the sections were incubated with horseradish peroxidase-labeled goat anti-rabbit immunoglobulin (cat. no. ab6721; Abcam) at a dilution of $1: 1,000$ at $37^{\circ} \mathrm{C}$ for $30 \mathrm{~min}$, and the reaction was visualized using 3,3'-diaminobenzidine. Eventually, the specimens were counterstained with Mayer's hematoxylin at room temperature for $1 \mathrm{~min}$. The images were photographed using an Olympus BX51 microscope with an Olympus DP-21 digital camera image system (Olympus Corporation). The primary antibody was replaced with non-immune rabbit serum (cat. no. AR0010; Boster, Wuhan, Hubei, China) which served as the negative control.

All samples were blindly inspected by two independent pathologists. Positive immunostaining was visualized as brown granules contained in the cytoplasm. The immunostaining of MMP-9 was scored on the scale of semi-quantitative assessment by evaluating the intensity and percentage of positively stained cells. The intensity of MMP-9 cytoplasmic staining was scored as follows: 0 , none; 1 , weak; 2 , moderate; and 3 , strong. The percentage scores were assigned, as follows: 

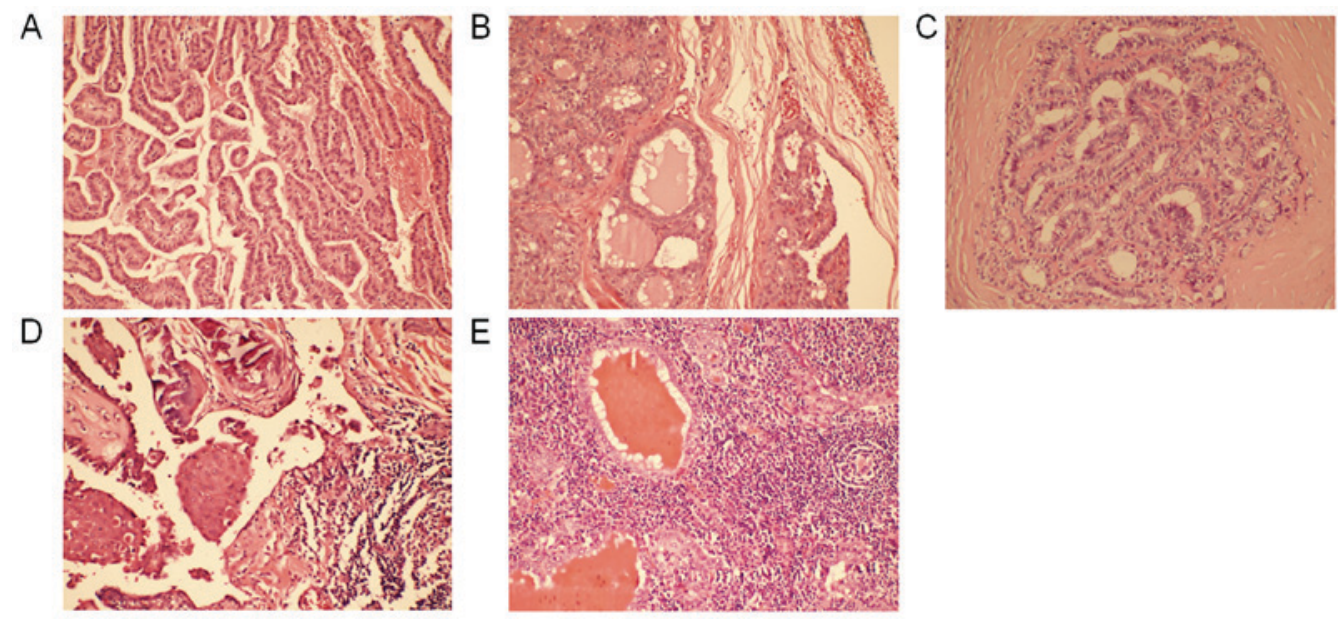

Figure 1. Pathological subtypes of PTC of the enrolled patients (hematoxylin and eosin staining; magnification, x200). (A) Classical PTC; (B) follicular variant of PTC; (C) tall cell variant; (D) diffuse sclerosing variant; (E) Warthin variant. PTC, papillary thyroid carcinoma.

$1, \leq 25 \% ; 2,26-50 \% ; 3,51-75 \%$; and $4,>75 \%$. These scores were multiplied to arrive at a final score ranging between 0 and 12 .

Statistical analysis. SPSS software (version 18.0; SPSS, Inc., Chicago, IL, USA) was used for statistical analysis. Data were presented as the means \pm standard deviation when data were normally distributed and the results were compared using independent student's t-test. Data expressed as median and interquartile ranges (IQRs) (25th percentile and 75th percentile) were compared using a Mann-Whitney nonparametric test. Categorical data were expressed as the number of patients and percentages, and the proportions were compared using $\chi^{2}$ test.

Receiver operating characteristic (ROC) curves were designed to estimate the diagnostic and prognostic value of MMP-9 in tissues, in terms of sensitivity, specificity, positive predictive value (PPV) and negative predictive value (NPV). The optimal cutoff values of tissue MMP-9 were identified according to the Youden index (26). The survival curves were plotted using the Kaplan-Meier method, and compared using log-rank test. Univariate Cox proportional hazard analysis was conducted to determine the risks of disease status and SPRD. Variables with significance $(\mathrm{P}<0.05)$ in univariate analyses were entered into the multivariable phase. Odds ratios (OR) and their $95 \%$ confidence intervals (CIs) were calculated to assess the independent contribution of each identified risk factor. $\mathrm{P}<0.05$ was considered to indicate a statistically significant difference.

\section{Results}

Clinicopathological characteristics of subjects. In the present study, 112 patients with PTC and 42 control subjects with BTN were included and separated into two groups. There was no significant difference in age between patients with PTC (45.77 \pm 10.03 years) and controls $(47.36 \pm 10.95$ years old $)$ $(\mathrm{P}>0.05)$. In addition, there were no significant differences in sex and proportion of subjects who were $\geq 45$ years old between these two groups $(\mathrm{P}>0.05)$. Numerous pathological subtypes of PTC were identified, including 96 cases of classical PTC, nine follicular variants of PTC, three tall cell variants, three diffuse sclerosing variants and one Warthin variant (Fig. 1A-E). The clinicopathological characteristics of these subjects are presented in Table I.

Diagnostic accuracy of MMP-9 for distinguishing PTC. MMP-9 was overexpressed in samples obtained from patients with PTC, when compared with control subjects with BTN (Fig. 2A-D). Furthermore, patients with PTC had a significantly higher IHC score of MMP-9 (median, 4.0; IQR, 2.0-8.0), when compared with control subjects (median, 1.0; IQR, 0.0-1.0) $(\mathrm{P}<0.001$, Fig. 2E).

The ROC curve for distinguishing PTC from BTN is presented in Fig. 3. MMP-9 scores yielded an area under the curve (AUC) of 0.842 (95\% CI, 0.776-0.908), with a sensitivity and specificity of 81.3 and $85.7 \%$, respectively, at a cutoff value of 2.0 points (Fig. 3). Furthermore, the PPV was $93.8 \%$, whereas the NPV was $63.2 \%$. In addition, the positive likelihood ratio was 5.690, whereas the negative likelihood ratio was 0.218 .

MMP-9 IHC expression in PTC with different invasive characteristics. Mann-Whitney test analysis was conducted to explore the association between MMP-9 and the invasive characteristics of PTC. The IHC expression of MMP-9 was greater if patients had central LNM (CLNM), lateral LNM (LLNM) or an advanced TNM stage (III+IV) (all $\mathrm{P}<0.05$; Table II). There was no difference in IHC MMP-9 expression in the presence or absence of capsule invasion, multifocality, extrathyroidal invasion, vascular invasion and distant metastasis (Table II).

Prognostic accuracy of MMP-9 for predicting disease status and SPRD. During the follow-up, patients with a disease status had a significantly higher MMP-9 score (median: 8, IQR: 6.0-9.0) than patients with a disease-free status (median: 3.0, IQR: 2.0-4.0; $\mathrm{P}<0.001)$. At a cutoff value of 6.0 points, MMP-9 yielded an AUC of 0.811 (95\% CI, 0.706-0.917), a sensitivity of $88.0 \%$ and a specificity of $78.2 \%$ for predicting disease-free status (Fig. 4A).

Patients with SPRD had a significantly higher MMP-9 score (median: 9.0; IQR: 7.0-10.5) than patients without SPRD (median: 4.0; IQR: 2.0-6.0) $(\mathrm{P}=0.010)$. Furthermore, when MMP-9 was employed to predict SPRD, an AUC of 
0.806 (95\% CI, 0.620-0.992) was obtained at a cutoff value of 8.0 points, with a sensitivity and specificity of 77.8 and $76.7 \%$, respectively (Fig. 4B).

Risk factors of disease status and SPRD. A univariate logistic regression analysis was performed to identify potential risk factors for predicting disease status. An age of $\geq 45$ years old, a tumor size of $>2 \mathrm{~cm}$, and the presence of CLNM, LLNM, vascular invasion, advanced TNM stage and MMP-9 $\geq 6$ points were identified as risk factors for the development of the disease status (all $\mathrm{P}<0.05)$. In the multivariate model, a tumor size $>2 \mathrm{~cm}$ (OR, 3.011; 95\% CI, 1.119-8.097; $\mathrm{P}=0.029)$ and MMP-9 $\geq 6$ points (OR, 12.210, 95\% CI, 3.404-43.798; $\mathrm{P}<0.001)$ were both independent risk factors for disease status in patients with PTC (Table III).

By univariate Cox regression analysis, a tumor size $>2 \mathrm{~cm}$, capsule invasion, CLNM, LLNM, extrathyroidal invasion, vascular invasion, distant metastasis, advanced TNM stage and an MMP-9 score of $\geq 8$ points were risk factors for SPRD (all $\mathrm{P}<0.05)$. Furthermore, multivariate analysis was performed to evaluate these univariate predictors, and the results revealed that vascular invasion (OR, 17.258; 95\% CI, 2.434-122.345; $\mathrm{P}=0.004)$ and an MMP-9 score of $\geq 8$ points (OR, 15.329; 95\% CI, 1.368-171.717; $\mathrm{P}=0.027$ ) were independent risk factors for SPRD (Table III).

Clinical outcomes stratified by MMP-9 scores. DFS time was shorter in PTC patients with an MMP-9 score of $\geq 6$ points (31.46 \pm 11.43 months), when compared to patients with an MMP-9 score of $<6$ points $(41.11 \pm 4.37$ months $)(\mathrm{P}<0.001)$. Similarly, PTC patients with an MMP-9 score of $\geq 8$ points had shorter survival time without SPRD (39.6 \pm 5.42 months), when compared with patients with an MMP-9 score of $<8$ points (41.70 \pm 2.34 months) $(\mathrm{P}=0.001)$. The Kaplan-Meier curves of survival demonstrated that patients with an MMP-9 score of $\geq 6$ points had lower cumulative DFS rates, when compared to patients with a score of $<6$ points $(\mathrm{P}<0.001)$. Furthermore, patients with an MMP-9 score of $\geq 8$ points had a lower cumulative rate of survival without SPRD, when compared to patients with a score of $<8$ points $(\mathrm{P}<0.001$, Fig. 5$)$.

\section{Discussion}

To the best of our knowledge, only a small number of studies regarding MMP-9 in thyroid tumorigenesis have been conducted, in comparison with tumors from other organs, including breast, ovarian, liver, lung and colon cancer, in which MMP-9 expression has already been extensively studied (27-31). In the present study, the IHC results revealed that MMP-9 expression was significantly higher in PTC tissues than in BTN specimens. In addition, the MMP-9 staining score was greater in the presence of CLNM, LLNM or in the case of advanced TNM stage. Furthermore, the MMP-9 score yielded good sensitivity and specificity for PTC diagnosis and prognostic prediction. A MMP-9 score of $\geq 6$ and $\geq 8$ indicated shortened DFS and survival without SPRD, respectively.

MMPs have essential roles in various biological functions, including cell proliferation, differentiation, ECM degradation and remodeling, angiogenesis and cell migration. Furthermore, the expression and activity of MMP-9 are elevated in numerous
Table I. Clinicopathological characteristics of patients, $\mathrm{n}(\%)$.

\begin{tabular}{|c|c|c|}
\hline Characteristic & PTC (n=112) & BTN $(n=42$ \\
\hline \multicolumn{3}{|l|}{ Age } \\
\hline$<45$ years old & $56(50.0)$ & $16(38.1)$ \\
\hline$\geq 45$ years old & $56(50.0)$ & $26(61.9)$ \\
\hline \multicolumn{3}{|l|}{ Sex } \\
\hline Female & $95(84.8)$ & $35(83.3)$ \\
\hline Male & $17(15.2)$ & $7(16.7)$ \\
\hline \multicolumn{3}{|l|}{ Tumor size } \\
\hline$\leq 2 \mathrm{~cm}$ & $81(72.3)$ & \\
\hline$>2 \mathrm{~cm}$ & $31(27.7)$ & \\
\hline \multicolumn{3}{|l|}{ Capsule invasion } \\
\hline No & $56(50.0)$ & \\
\hline Yes & $56(50.0)$ & \\
\hline \multicolumn{3}{|l|}{ Multifocality } \\
\hline Unifocal & $69(61.6)$ & \\
\hline Multifocal & $43(38.4)$ & \\
\hline \multicolumn{3}{|l|}{ Nodal status } \\
\hline N0 & $49(43.8)$ & \\
\hline N1a & $42(37.5)$ & \\
\hline N1b & $21(18.7)$ & \\
\hline \multicolumn{3}{|c|}{ Extrathyroidal invasion } \\
\hline Negative & $78(69.6)$ & \\
\hline Microscopic & $25(22.3)$ & \\
\hline Macroscopic & $9(8.0)$ & \\
\hline \multicolumn{3}{|c|}{ Vascular invasion } \\
\hline No & $107(95.5)$ & \\
\hline Yes & $5(4.5)$ & \\
\hline
\end{tabular}

Distant metastasis

$\begin{array}{lc}\text { No } & 109(97.3) \\ \text { Yes } & 3(2.7) \\ \text { TNM stage } & \\ \text { I+II } & 77(68.8) \\ \text { III+IV } & 35(31.3)\end{array}$

BTN, benign thyroid nodule; PTC, papillary thyroid carcinoma; TNM, tumor-node-metastasis. ${ }^{a}$ Analyzed by $\chi^{2}$ test.

types of human cancer (32-34). In addition, the IHC positive staining of MMP-9 has been reported in 57.0-92.4\% of patients with PTC $(3,24)$. In the present study, MMP-9 was expressed at low or almost undetectable levels in BTN specimens (median: 1.0; IQR: 0.0-1.0). Conversely, overexpression of MMP-9 (median: 4.0; IQR: 2.0-8.0) was detected in PTC tissues, and was associated with tumor malignancy. The majority of MMPs are secreted in an inactive form, and can be proteolytically activated by extracellular proteinase, which is critically implicated in carcinogenesis (35). The present findings revealed that the MMP-9 IHC score may be useful for the differential diagnosis of PTC from BTN, with a sensitivity and specificity of 81.3 and $85.7 \%$, respectively, and at a cutoff value of 2.0 points. Consistently, Meng et al demonstrated that the 


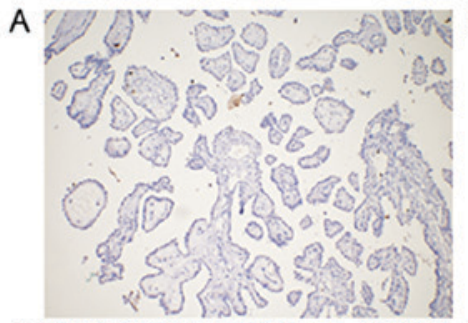

C

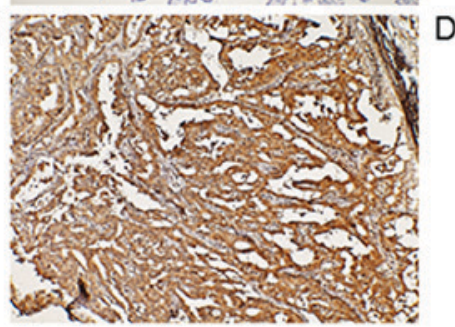

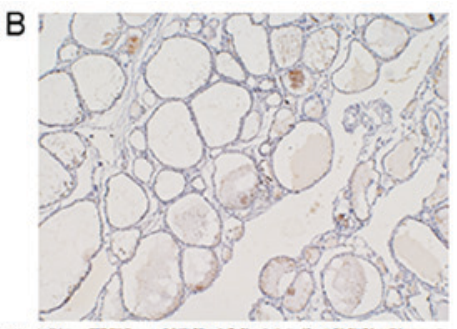

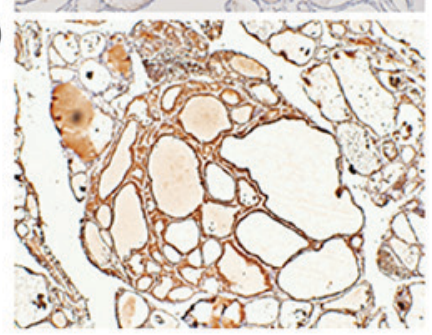

E

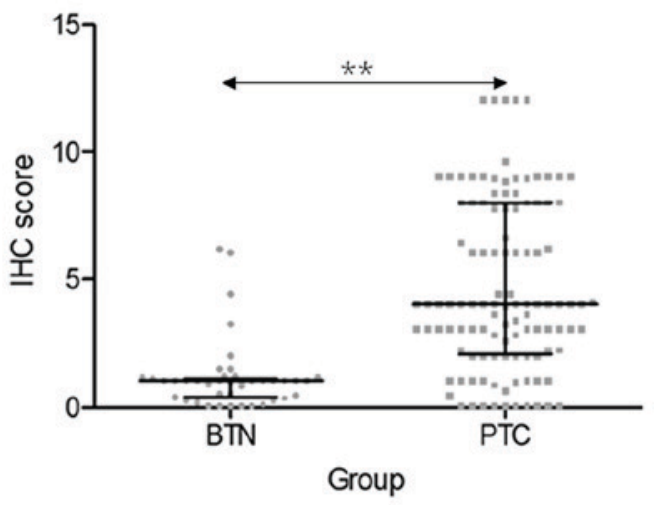

Figure 2. Immunohistochemical expression levels of MMP-9. MMP-9 immunostaining was performed in tumors obtained from patients with PTC, and was compared with specimens obtained from patients with BTN. Positive staining was defined as brown-yellow particles or tan-brown particles in the cytoplasm (magnification, x100). (A) PTC with negative staining (score 0); (B) BTN with negative staining (score 0); (C) PTC with positive staining (score 12); (D) BTN with positive staining (score 12). (E) Immunostaining of MMP-9 was scored on the scale of semi-quantitative assessment by evaluating the intensity and percentage of positive cells. The black central horizontal lines indicate the median, and the error bars (upper and lower horizontal lines) represent the 75th percentile and 25th percentile, respectively, ${ }^{* *} \mathrm{P}<0.001$. Data were analyzed using Mann-Whitney nonparametric test. BTN, benign thyroid tumors; IHC, immunohistochemistry; MMP-9, matrix metalloproteinase-9; PTC, papillary thyroid carcinoma.

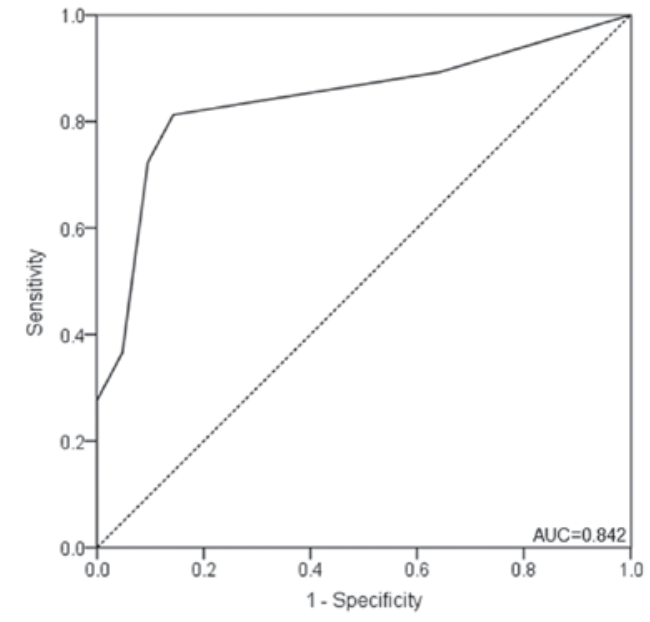

Figure 3. Receiver operating characteristic curve of immunohistochemical matrix metalloproteinase- 9 staining for the diagnosis of papillary thyroid carcinoma.

immunohistochemical staining of MMP-9 yields a sensitivity and specificity of 92.4 and $80 \%$, respectively, for PTC diagnosis from BTN (24), thus suggesting a role for MMP-9 in PTC diagnosis. In addition, vasculature is important for tumor size and growth; if the tumor vasculature is not developed, tumors are restricted in size within a tissue-diffusion distance of 0.2-2.0 mm (36). MMP-9 has been demonstrated to participate in angiogenesis, as a result, MMP-9 overexpression is likely to be associated with a larger tumor size and is the dependent risk factor for disease status and $\operatorname{SPRD}(23,37)$. Above all, MMP-9 is associated with the prognosis of PTC. MMP-9 has been demonstrated to participate in the switch from vascular quiescence to angiogenesis, which is a crucial process required for persistent tumor growth (38). The MMP-dependent release of growth factors, including VEGF and FGF, stimulates the progressive growth of tumor cells (39). This may explain the present finding that stronger MMP-9 immunostaining was likely to be associated with a larger tumor size $(>2 \mathrm{~cm})$. Furthermore, tumor size $(>2 \mathrm{~cm})$ was dependent predictor for disease status and SPRD, indicating that MMP-9 was correlated with the prognosis of PTC.

Invasion and metastasis substantially contribute to cancer-associated mortality. The disruption of basement membranes is a crucial step in the process of cancer invasion to surrounding tissues and of metastasis to distant organs. The role of MMP-9 is to degrade type IV collagen, which is the main structural component of the basement membrane, and is of particular importance in tumor cell migration (40). MMP-9-mediated tumor angiogenesis creates a microenvironment favorable to tumor cell invasion by promoting gas exchange and supplying nutrients (41). Furthermore, MMP-9 expression has been reported to be associated with microvessel density in colorectal carcinoma (42). In addition, MMP-9 is an essential mediator of epithelial-mesenchymal transition, which is a key step in tumor progression and metastasis (43). The association between MMP-9 overexpression and the invasiveness of cancer has been reported in numerous studies (32,44-47). In patients with PTC, positive MMP-9 staining is correlated with lymphatic spreading and the degree of tumor infiltration (3). Consistent with these findings, the IHC score of MMP-9 was greater in the presence of CLNM, LLNM or advanced TNM stage, indicating that MMP-9 may be used as a predictive biomarker of aggressive PTC behaviors.

The association between MMP-9 expression and malignant neoplasms has garnered much interest, and MMP-9 may be considered a predictor of patient prognosis. It has been reported that elevated MMP-9 expression, as an independent risk factor, is associated with advanced tumor stage and shortened survival in various types of cancer $(47,48)$. In the present study, MMP-9 expression in PTC samples was revealed to represent an independent risk factor for predicting the presence of disease and SPRD following thyroidectomy. Subsequently, high 
Table II. MMP-9 immunohistochemistry expression differences in the groups stratified according to clinicopathological characteristics.

Variable

MMP-9 score $^{\mathrm{a}}$

P-value

Tumor size (>2 cm vs. $\leq 2 \mathrm{~cm}$ )

$6.0(3.0-9.0)$ vs. $4.0(2.0-6.0)$

0.053

Capsule invasion (yes vs. no)

$4.0(2.0-9.0)$ vs. $3.0(2.0-6.0)$

0.090

Multifocality (yes vs. no)

$4.0(1.0-8.0)$ vs. $4.0(3.0-8.0)$

0.499

Central lymph node metastasis (yes vs. no)

$7.0(3.0-9.0)$ vs. $3.0(2.0-4.0)$

0.002

Lateral lymph node metastasis (yes vs. no)

$8.0(5.0-9.0)$ vs. $3.0(2.0-4.0)$

$<0.001$

Extrathyroidal invasion (yes vs. no)

$5.0(3.0-9.0)$ vs. $4.0(2.0-6.0)$

0.065

Vascular invasion (yes vs. no)

$6.0(1.0-9.0)$ vs. $4.0(2.0-8.0)$

0.691

Distant metastasis (yes vs. no)

$3.0(2.0-6.0)$ vs. $4.0(2.0-8.0)$

0.721

TNM stage (III+IV vs. I+II)

$8.0(3.0-9.0)$ vs. $3.0(2.0-6.0)$

0.004

MMP-9, matrix metalloproteinase-9; TNM, tumor-node-metastasis. ${ }^{a}$ Data are presented as the median (25th percentile and 75th percentile). ${ }^{\mathrm{b}}$ Analyzed by Mann-Whitney test. Bold indicates a significant difference.
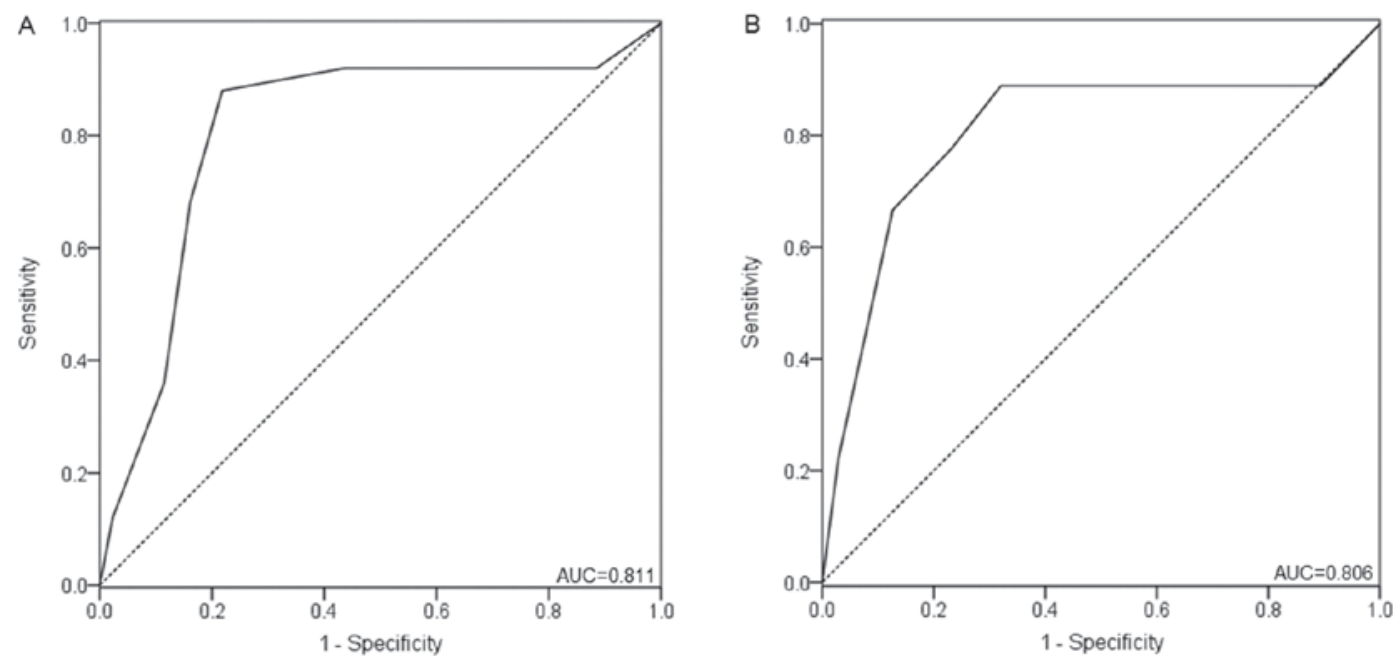

Figure 4. Receiver operating characteristic curves of the immunohistochemical matrix metalloproteinase-9 staining for predicting the prognosis of patients with papillary thyroid carcinoma. (A) Disease-free status; (B) structurally persistent/recurrent disease.
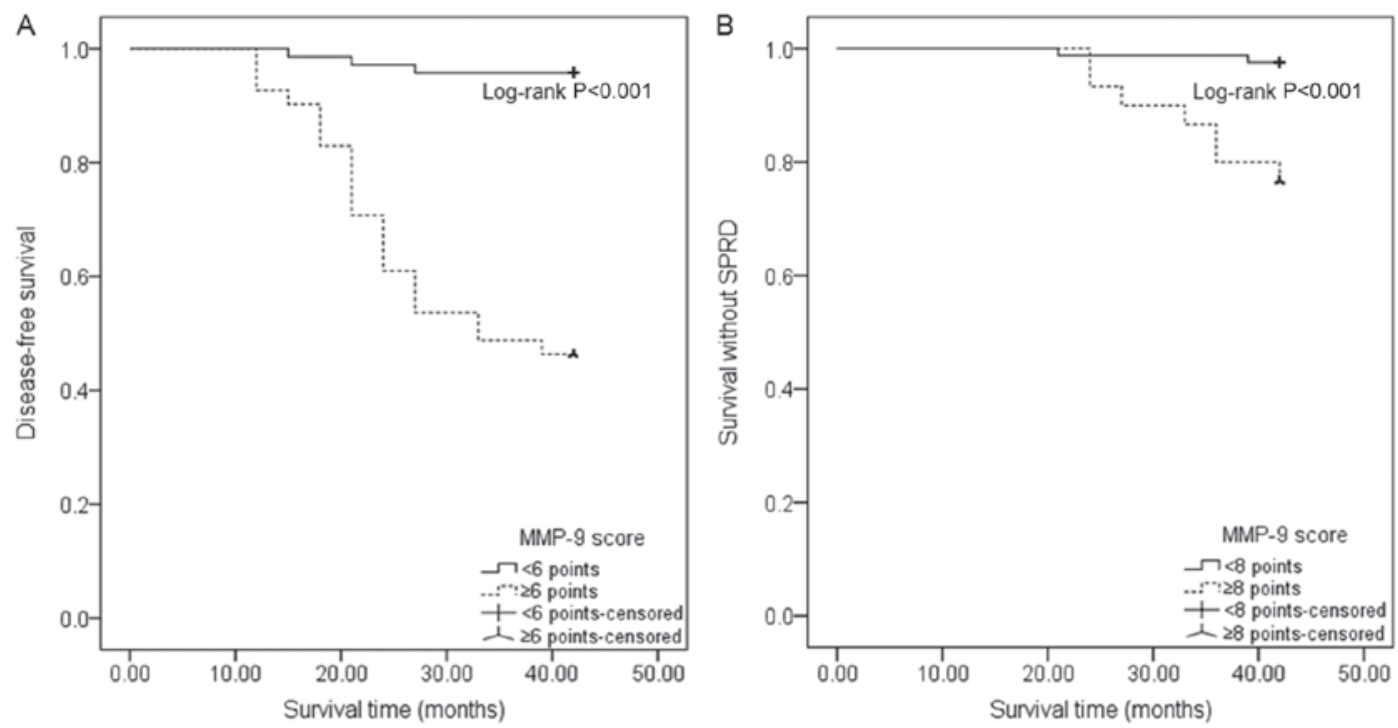

Figure 5. Survival curves of patients with papillary thyroid carcinoma by MMP-9 stratification. (A) Cumulative disease-free survival rate for patients with MMP- $9 \geq 6$ points vs. $<6$ points. (B) Cumulative survival rate without SPRD for patients with MMP-9 $\geq 8$ points vs. $<8$ points. MMP-9, matrix metalloproteinase-9; SPRD, structurally persistent/recurrent disease. 
Table III. Risk factors of disease status and SPRD in patients with PTC, as determined by Cox regression model.

A, Disease status

\begin{tabular}{|c|c|c|c|c|}
\hline \multirow[b]{2}{*}{ Characteristic } & \multicolumn{2}{|c|}{ Univariate analysis } & \multicolumn{2}{|c|}{ Multivariate analysis } \\
\hline & OR $(95 \% \mathrm{CI})$ & P-value & OR $(95 \% \mathrm{CI})$ & P-value \\
\hline Age $(\geq 45$ years old vs. $<45$ years old $)$ & $4.528(1.698-12.072)$ & 0.003 & $2.269(0.677-7.600)$ & 0.184 \\
\hline Sex (male vs. female) & $1.511(0.567-4.027)$ & 0.409 & & \\
\hline BMI $\left(\geq 25 \mathrm{~kg} / \mathrm{m}^{2}\right.$ vs. $\left.<25 \mathrm{~kg} / \mathrm{m}^{2}\right)$ & $1.731(0.765-3.918)$ & 0.188 & & \\
\hline Tumor size $(>2 \mathrm{~cm}$ vs. $\leq 2 \mathrm{~cm})$ & $3.353(1.528-7.358)$ & 0.003 & $3.011(1.119-8.097)$ & 0.029 \\
\hline Capsule invasion (yes vs. no) & $1.583(0.711-3.524)$ & 0.261 & & \\
\hline Multifocality (yes vs. no) & $1.364(0.619-3.005)$ & 0.441 & & \\
\hline CLNM (yes vs. no) & $3.915(1.688-9.082)$ & 0.001 & $1.990(0.729-5.430)$ & 0.179 \\
\hline LLNM (yes vs. no) & $3.932(1.780-8.688)$ & 0.001 & $0.730(0.256-2.085)$ & 0.557 \\
\hline Extrathyroidal invasion (yes vs. no) & $2.041(0.916-4.545)$ & 0.081 & & \\
\hline Vascular invasion (yes vs. no) & $3.446(1.029-11.456)$ & 0.045 & $2.384(0.655-8.678)$ & 0.188 \\
\hline Distant metastasis (yes vs. no) & $3.354(0.788-14.273)$ & 0.101 & & \\
\hline TNM stage (III+IV vs. I+II) & $2.632(1.200-5.773)$ & 0.016 & $0.465(0.151-1.431)$ & 0.182 \\
\hline MMP-9 score ( $\geq 6$ points vs. $<6$ points) & $16.665(4.975-55.826)$ & $<0.001$ & $12.210(3.404-43.798)$ & $<0.001$ \\
\hline
\end{tabular}

B, SPRD

Univariate analysis

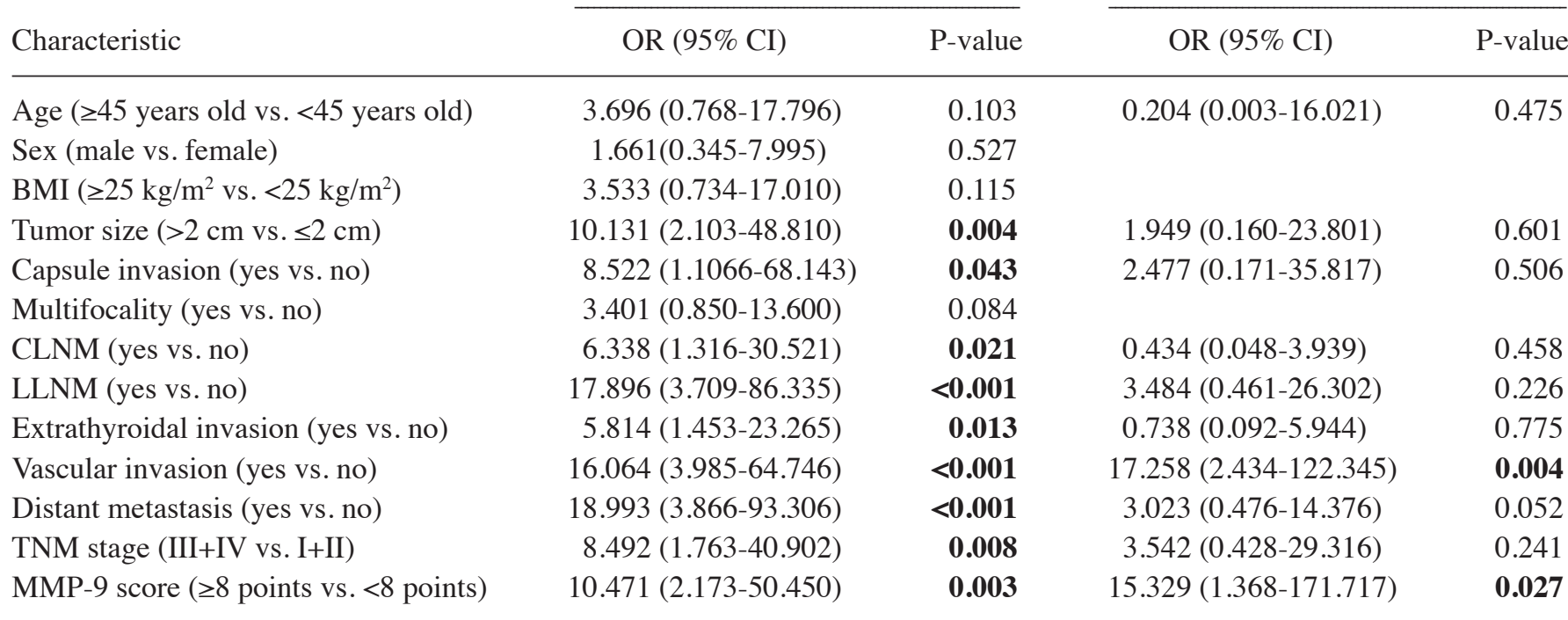

BMI, body mass index; CLNM, central lymph node metastasis; LLNM, lateral lymph node metastasis; PTC, papillary thyroid carcinoma; SPRD, structurally persistent disease/recurrent disease; TNM, tumor-node-metastasis. Bold indicates a significant difference. The Univariate Cox proportional hazard analysis was conducted to determine the risks of disease status and SPRD. Variables with significance $(\mathrm{P}<0.05)$ in univariate analyses were entered into the multivariable phase.

MMP-9 expression was associated with shortened DFS and survival without SPRD in patients with PTC. These results are consistent with a previous study, which reported that MMP-9 is an independent prognostic factor for predicting the worse outcome in patients with PTC following radiofrequency ablation (49). In addition, Lin et al revealed that median event-free survival was shorter in patients with T-cell acute lymphoblastic leukemia with the CT+TT genotype of MMP-9-1562C>T, when compared to patients with the CC genotype, suggesting that MMP-9 genetic polymorphism may affect tumor progression and prognosis (50). Therefore, the genetic characteristics of MMP-9 and its association with PTC prognosis require further investigation.

The present study differs from previous studies in many ways. To the best of our knowledge, it is the first to carefully assess the diagnostic and prognostic values of MMP-9 immunostaining in patients with PTC and after thyroidectomy. PTC is a more common malignancy in women than in men (51); however, male patients with PTC are common in China (52). The sex and age were well matched between the BTN group 
and PTC group, in order to minimize any influences caused by age and sex. In addition, the relationships between MMP-9 and SPRD, and survival without SPRD, were examined, which has rarely been reported in previous studies investigating the predictive role of MMP-9 in PTC prognosis. Finally, previous studies have focused on other proteins, and MMP-9 has only been examined with regards to a few aspects of PTC prognosis $(1,3,22,23)$. The present study systematically analyzed the diagnostic and prognostic roles of immunohistochemical MMP-9 in patients with PTC, and identified it as an independent risk factor for disease status and survival. Consequently, the present study may provide detailed evidence for deciding the usefulness of MMP-9 in PTC prediction.

The present study presented numerous limitations. Firstly, this study was a single-center, retrospective cohort analysis with a relatively small sample size. In addition, a longer follow-up period is required to further analyze the relationship between alterations in MMP-9 and the prognosis of patients. Secondly, IHC staining is only a semi-quantitative method for testing MMP-9 protein levels. Quantitative measurements of MMP-9 expression levels and protein activation should be conducted to validate these findings. Thirdly, selection bias at the time of study enrollment may have occurred. Notably, some patients with small PTC lesions or BTNs who refused dissection were not recruited in the study. Finally, although there were five subtypes of PTC in the present study, the number of patients with each of them was very small; therefore, all PTC subtypes were added and analyzed as a whole.

In conclusion, the present study demonstrated that immunohistochemical MMP-9 expression was markedly increased in PTC. In addition, elevated MMP-9 expression was associated with central lymph node metastasis, lateral lymph node metastasis, advanced TNM stage and shortened patient survival. Therefore, the assessment of MMP-9 expression in thyroid carcinoma samples may represent a novel tool for PTC diagnosis and prognostic prediction.

\section{Acknowledgements}

Not applicable.

\section{Funding}

The present study was supported by the Special Project for Health in Jilin Province (grant nos. 2018SCZWSZX-039 and 2018SCZWSZX-050), Projects of the Education Department of Jilin Province (grant nos. 20150125, 2016453 and 2016482), and the Project of the Health Department of Jilin Province (grant no. 20150125).

\section{Availability of data and materials}

The datasets used and/or analyzed during the current study are available from the corresponding author on reasonable request.

\section{Authors' contributions}

XL, JX and CS analyzed and interpreted the patient data on papillary thyroid diseases and BTNs. DX and NZ performed the histological examination of the thyroid tissues. HH was the major contributor in designing the experiment and writing the manuscript. DZ, HY, WL and GC prepared the figures and conducted the statistical analyses. All authors read and approved the final manuscript.

\section{Ethics approval and consent to participate}

The study methodology was approved by the Ethics Committee of the First Hospital of Jilin University. Patients provided informed consent.

\section{Patient consent for publication}

All participants provided written informed consent prior to participation.

\section{Competing interests}

The authors declare that they have no competing interests.

\section{References}

1. Zhang Y, Luo YK, Zhang MB, Li J, Li CT, Tang J and Li JL: Values of ultrasound features and MMP-9 of papillary thyroid carcinoma in predicting cervical lymph node metastases. Sci Rep 7: 6670, 2017.

2. Šelemetjev S, Đoric I, Paunovic I, Tatic S and Cvejic D: Coexpressed high levels of VEGF-C and active MMP-9 are associated with lymphatic spreading and local invasiveness of papillary thyroid carcinoma. Am J Clin Pathol 146: 594-602, 2016.

3. Huang LL, Wang Z, Cao CJ, Ke ZF, Wang F, Wang R, Luo CQ, Lu X and Wang LT: AEG-1 associates with metastasis in papillary thyroid cancer through upregulation of MMP2/9. Int J Oncol 51: 812-822, 2017.

4. Mazzaferri EL and Jhiang SM: Long-term impact of initial surgical and medical therapy on papillary and follicular thyroid cancer. Am J Med 97: 418-428, 1994.

5. Zhang M, Wu W, Gao M and Fei Z: MicroRNA-451 as a prognostic marker for diagnosis and lymph node metastasis of papillary thyroid carcinoma. Cancer Biomark 19: 437-445, 2017.

6. Vuong HG, Duong UN, Altibi AM, Ngo HT, Pham TQ, Tran HM, Gandolfi G and Hassell L: A meta-analysis of prognostic roles of molecular markers in papillary thyroid carcinoma. Endocr Connect 6: R8-R17, 2017.

7. Kim Y, Kim MH, Jeon S, Kim J, Kim C, Bae JS and Jung CK: Prognostic implication of histological features associated with EHD2 expression in papillary thyroid carcinoma. PLoS One 12: e0174737, 2017.

8. Castro MG, Campos LE, Rodriguez YI and Alvarez SE: In vitro methods to study the modulation of migration and invasion by sphingosine-1-phosphate. Methods Mol Biol 1697: 117-131, 2018.

9. Edatt L, Maurya AK, Raji G, Kunhiraman H and Kumar SVB: MicroRNA106a regulates matrix metalloprotease 9 in a sirtuin-1 dependent mechanism. J Cell Physiol 233: 238-248, 2018.

10. Zhou Q, Guo X and Choksi R: Activation of focal adhesion kinase and Src mediates acquired sorafenib resistance in A549 human lung adenocarcinoma xenografts. J Pharmacol Exp Ther 363: 428-443, 2017.

11. Zhou G, Peng F, Zhong Y, Chen Y, Tang M and Li D: Rhein suppresses matrix metalloproteinase production by regulating the Rac1/ROS/MAPK/AP-1 pathway in human ovarian carcinoma cells. Int J Oncol 50: 933-941, 2017.

12. Wang L and Xue GB: Catalpol suppresses osteosarcoma cell proliferation through blocking epithelial-mesenchymal transition (EMT) and inducing apoptosis. Biochem Biophys Res Commun 495: 27-34, 2018.

13. Lai XX, Li G, Lin B and Yang H: Interference of Notch 1 inhibits the proliferation and invasion of breast cancer cells: Involvement of the $\beta$-catenin signaling pathway. Mol Med Rep 17: 2472-2478, 2018. 
14. Bai XY, Li S, Wang M, Li X, Yang Y, Xu Z, Li B, Li Y, Xia K, Chen $\mathrm{H}$ and $\mathrm{Wu} \mathrm{H}$ : Krüppel-like factor 9 down-regulates matrix metalloproteinase 9 transcription and suppresses human breast cancer invasion. Cancer Lett 412: 224-235, 2018.

15. Zhu N, Si M, Yang N, Jing Y, Fu Y, Zhao X, Lin Z and Yang G: Overexpression of RAS-association domain family 6 (RASSF6) inhibits proliferation and tumorigenesis in hepatocellular carcinoma cells. Oncol Res 25: 1001-1008, 2017.

16. Sun Y, Chen Y, Li S, Lei Y, Xu D, Jiang N, Zhang Y, Cao J and Ke Z: NanoVelcro-captured CTC number concomitant with enhanced serum levels of MMP7 and MMP9 enables accurate prediction of metastasis and poor prognosis in patients with lung adenocarcinoma. Int J Nanomedicine 12: 6399-6412, 2017.

17. Bergers G, Brekken R, McMahon G, Vu TH, Itoh T, Tamaki K, Tanzawa K, Thorpe P, Itohara S, Werb Z and Hanahan D: Matrix metalloproteinase-9 triggers the angiogenic switch during carcinogenesis. Nat Cell Biol 2: 737-744, 2000.

18. Ucuzian AA, Gassman AA, East AT and Greisler HP: Molecular mediators of angiogenesis. J Burn Care Res 31: 158-175, 2010.

19. Malemud CJ: Matrix metalloproteinases (MMPs) in health and disease: An overview. Front Biosci 11: 1696-1701, 2006.

20. Yang XZ, Cui SZ, Zeng LS, Cheng TT, Li XX, Chi J, Wang R, Zheng XF and Wang HY: Overexpression of Rab1B and MMP9 predicts poor survival and good response to chemotherapy in patients with colorectal cancer. Aging (Albany NY) 9: 914-931, 2017.

21. Xue Q, Cao L, Chen XY, Zhao J, Gao L, Li SZ and Fei Z: High expression of MMP9 in glioma affects cell proliferation and is associated with patient survival rates. Oncol Lett 13: 1325-1330, 2017.

22. Luo D, Chen H, Li X, Lu P, Long M, Peng X, Lin S, Tan L, Zhu Y, Ouyang $\mathrm{N}$ and Li H: Activation of the ROCK1/MMP-9 pathway is associated with the invasion and poor prognosis in papillary thyroid carcinoma. Int J Oncol 51: 1209-1218, 2017.

23. Wang N, Jiang R, Yang JY, Tang C, Yang L, Xu M, Jiang QF and Liu ZM: Expression of TGF- $\beta 1$, SNAI1 and MMP-9 is associated with lymph node metastasis in papillary thyroid carcinoma. J Mol Histol 45: 391-399, 2014.

24. Meng XY, Zhang Q, Li Q, Lin S and Li J: Immunohistochemical levels of cyclo-oxygenase-2, matrix metalloproteinase-9 and vascular endothelial growth factor in papillary thyroid carcinoma and their clinicopathological correlations. J Int Med Res 42: 619-627, 2014.

25. Lee JJ, Wang TY, Liu CL, Chien MN, Chen MJ, Hsu YC, Leung $\mathrm{CH}$ and Cheng SP: Dipeptidyl peptidase IV as a prognostic marker and therapeutic target in papillary thyroid carcinoma. J Clin Endocrinol Metab 102: 2930-2940, 2017.

26. Pang L, Zhang N, Xia Y, Wang D, Wang G and Meng X: Serum APN/CD13 as a novel diagnostic and prognostic biomarker of pancreatic cancer. Oncotarget 7: 77854-77864, 2016.

27. Sun XF, Shao YB, Liu MG, Chen Q, Liu ZJ, Xu B, Luo SX and Liu H: High-concentration glucose enhances invasion in invasive ductal breast carcinoma by promoting Glut1/MMP2/MMP9 axis expression. Oncol Lett 13: 2989-2995, 2017.

28. Zhou G, Peng F, Zhong Y, Chen Y, Tang M and Li D: Rhein suppresses matrix metalloproteinase production by regulating the Rac1/ROS/MAPK/AP-1 pathway in human ovarian carcinoma cells. Int J Oncol 50: 933-941, 2017.

29. Zhu N, Si M, Yang N, Jing Y, Fu Y, Zhao X, Lin Z and Yang G: Overexpression of RAS-association domain family 6 (RASSF6) inhibits proliferation and tumorigenesis in hepatocellular carcinoma cells. Oncol Res 25: 1001-1008, 2017.

30. Zhu B, Yang J, Zhang P, Shen L, Li X and Li J: Safety and effectiveness of localized lung resection combined with neoadjuvant chemotherapy in the treatment of stage I-II non-small cell lung cancer. Oncol Lett 13: 2344-2348, 2017.

31. Zhang R, Zhao J, Xu J, Jiao DX, Wang J, Gong ZQ and Jia JH: Andrographolide suppresses proliferation of human colon cancer SW620 cells through the TLR4/NF- $\mathrm{B} / \mathrm{MMP}-9$ signaling pathway. Oncol Lett 14: 4305-4310, 2017.

32. Ricci S, Guadagno E, Bruzzese D, Del Basso De Caro M, Peca C, Sgulò FG, Maiuri F and Di Carlo A: Evaluation of matrix metalloproteinase type IV-collagenases in serum of patients with tumors of the central nervous system. J Neurooncol 131: 223-232, 2017.

33. Reiner AT, Tan S, Agreiter C, Auer K, Bachmayr-Heyda A, Aust S, Pecha N, Mandorfer M, Pils D, Brisson AR, et al: EV-associated MMP9 in high-grade serous ovarian cancer is preferentially localized to annexin V-binding EVs. Dis Markers 2017: 9653194, 2017.
34. Skerenova M, Mikulova V, Capoun O, Zima T and Tesarova P: Circulating tumor cells and serum levels of MMP-2, MMP-9 and VEGF as markers of the metastatic process in patients with high risk of metastatic progression. Biomed Pap Med Fac Univ Palacky Olomouc Czech Repub 161: 272-280, 2017.

35. Kessenbrock K, Plaks V and Werb Z: Matrix metalloproteinases: Regulators of the tumor microenvironment. Cell 141: 52-67, 2010

36. Fang J, Shing Y, Wiederschain D, Yan L, Butterfield C, Jackson G, Harper J, Tamvakopoulos G and Moses MA: Matrix metalloproteinase-2 is required for the switch to the angiogenic phenotype in a tumor model. Proc Natl Acad Sci USA 97: 3884-3889, 2000.

37. He J, Shen N and Huang X: Thyroid carcinoma cells produce PLGF to enhance metastasis. Tumour Biol 36: 8601-8607, 2015.

38. Yu Q and Stamenkovic I: Cell surface-localized matrix metalloproteinase-9 proteolytically activates TGF-beta and promotes tumor invasion and angiogenesis. Genes Dev 14: 163-176, 2000.

39. Chang $\mathrm{C}$ and Werb Z: The many faces of metalloproteases: Cell growth, invasion, angiogenesis and metastasis. Trends Cell Biol 11: S37-S43, 2001.

40. Zheng $\mathrm{H}$ and Liu JF: Studies on the relationship between P13K/AKT signal pathway-mediated MMP-9 gene and lung cancer. Eur Rev Med Pharmacol Sci 21: 753-759, 2017.

41. DeryuginaEIandQuigleyJP:Tumorangiogenesis:MMP-mediated induction of intravasation- and metastasis-sustaining neovasculature. Matrix Biol 44-46: 94-112, 2015

42. Wu XL, Xue J, Wang LK, Yang DD, Qu M, Guo F, Sun GY, Han L and Yang RM: Expressions of inhibitors of DNA binding-1 and matrix metalloproteinase-9 in colorectal adenocarcinoma tissues and their correlations with microvessel density. Zhongguo Yi Xue Ke Xue Yuan Xue Bao 38: 696-701, 2016.

43. Bai X, Li YY, Zhang HY, Wang F, He HL, Yao JC, Liu L and Li SS: Role of matrix metalloproteinase-9 in transforming growth factor- $\beta 1$-induced epithelial-mesenchymal transition in esophageal squamous cell carcinoma. Onco Targets Ther 10: 2837-2847, 2017

44. Zhu XM and Sun WF: Association between matrix metalloproteinases polymorphisms and ovarian cancer risk: A meta-analysis and systematic review. PLoS One 12: e0185456, 2017.

45. Hsu CC, Huang SF, Wang JS, Chu WK, Nien JE, Chen WS and Chow SE: Interplay of N-cadherin and matrix metalloproteinase 9 enhances human nasopharyngeal carcinoma cell invasion. BMC Cancer 16: 800, 2016.

46. Rašić I, Rašić A, Akšamija G, Radović S and Šehović N: The association between the serum levels of matrix metalloproteinase 9 and colorectal cancer. Med Glas (Zenica) 14: 229-235, 2017.

47. Li H, Qiu Z, Li F and Wang C: The relationship between MMP-2 and MMP-9 expression levels with breast cancer incidence and prognosis. Oncol Lett 14: 5865-5870, 2017.

48. Lee CY, Shim HS, Lee S, Lee JG, Kim DJ and Chung KY: Prognostic effect of matrix metalloproteinase-9 in patients with resected non small cell lung cancer. J Cardiothorac Surg 10: 44 2015.

49. He J, Liu G, Shao K, Shen X and Chen L: Serum contents of matrix metalloproteinase- 2 and 9 are correlated with the prognosis of papillary thyroid carcinoma after ultrasound-guided radiofrequency ablation. Biomed Res 28: 6711-6716, 2017.

50. Lin CM, Zeng YL, Xiao M, Mei XQ, Shen LY, Guo MX, Lin ZY, Liu QF and Yang T: The relationship between MMP-2 $-1306 \mathrm{C}>\mathrm{T}$ and MMP-9-1562C $>\mathrm{T}$ polymorphisms and the risk and prognosis of T-cell acute lymphoblastic leukemia in a chinese population: A case-control study. Cell Physiol Biochem 42: 1458-1468, 2017.

51. Aschebrook-Kilfoy B, Ward MH, Sabra MM and Devesa SS: Thyroid cancer incidence patterns in the United States by histologic type, 1992-2006. Thyroid 21: 125-134, 2011.

52. Yan HX, Pang P, Wang FL, Tian W, Luo YK, Huang W, Yang GQ, Jin N, Zang L, Du J, et al: Dynamic profile of differentiated thyroid cancer in male and female patients with thyroidectomy during 2000-2013 in China: A retrospective study. Sci Rep 7: 15832, 2017.

This work is licensed under a Creative Commons Attribution-NonCommercial-NoDerivatives 4.0 International (CC BY-NC-ND 4.0) License. 